\title{
Abducens Nerve (VI) Injury Caused by Halo-Femoral Traction: A Case Report
}

\author{
Alaa A. Abd-Elsayed ${ }^{\mathrm{a}, \mathrm{c}}$, Paul Samuels ${ }^{\mathrm{b}}$, George Istaphanous ${ }^{\mathrm{b}}$
}

\begin{abstract}
Stretch and traction on cranial nerves during surgery may cause localized ischemia or change of position of the cranial nerve leading to subsequent dysfunction. Cranial nerve palsies (VI, IX, X and XII) are the commonly affected nerves. Here we report a cranial nerve VI palsy from a halo-femoral suspension following a posterior spinal fusion. We present an 11-year-old girl with idiopathic scoliosis who was admitted for surgical correction. She had no neurological problems preoperatively. The initial video-assisted thoracoscopic anterior release and cranial bifemoral traction were uneventful. Postoperatively, ten-pound weights were added at the head and another ten were added for counter-traction. Weights were added daily in 5-pound increments. Postoperative day 2, the patient complained of "double vision" which resolved upon decreasing the traction weight only by 5 pounds. She underwent an uneventful posterior spinal fusion one week later with a total intravenous anesthesia technique; motor evoked potentials were adequate throughout the procedure. One day later, she again developed left sixth cranial nerve palsy with inability to gaze laterally. There were no other neurological deficits, headaches or even double vision. Neurology and ophthalmology consultations excluded hypoxia/hypoperfusion as possible etiologies. The femoral pins and occipital frame were removed on day 3 in order to obtain imaging and also to remove a potential source of infection. The left lateral rectus palsy was noted to improve that same day. Radiological imaging results were ultimately normal. There is a need for frequent neurological examinations in patients in halo-tractions and prompt removal of the instrument at the first sign of eye problems.
\end{abstract}

Manuscript accepted for publication July 22, 2011

${ }^{a}$ Anesthesiology, University of Cincinnati, Cincinnati, Ohio, USA

${ }^{\mathrm{b}}$ Anesthesiology, Cincinnati Children's Hospital Medical Center,

University of Cincinnati, Cincinnati, Ohio, USA

${ }^{\mathrm{C} C o r r e s p o n d i n g ~ a u t h o r: ~ A l a a ~ A . ~ A b d-E l s a y e d, ~ A n e s t h e s i o l o g y, ~ U n i v e r s i t y ~}$ of Cincinnati, 231 Albert Sabin Way, Cincinnati, Ohio 45267-0531, USA.Email: alaaawny@hotmail.com
Keywords: Cranial nerve palsy; Abducens nerve; Scoliosis; Halofemoral traction; Posterior spinal fusion

\section{Introduction}

The first reported complications of the halo-traction device in 1968 by Nickel et al [1] included, infection, subdural abscesses, paraplegia, deep venous thrombosis and death. Cranial nerve palsies (VI, IX, X and XII) have also been reported in the past in the orthopedic but not in the anesthesia literature [2-5]. Stretch and traction forces can cause localized ischemia or changes in the position of the nerve. Here we report unusual cranial nerve VI (abducens nerve) palsy from a halo-femoral suspension following a posterior spinal fusion. Anesthesiologists should be familiar with such complications as the devices are typically installed in the operating room, and weights maybe manipulated during the surgery. Also patients will present to the operating rooms with such devices for multiple-staged procedures.

\section{Case Report}

An otherwise-healthy 11-year-old girl with idiopathic scoliosis was admitted for a two-staged, anterior release and posterior spinal fusion procedure. She was an active girl with no neurological problems secondary to her 85 degrees of scoliosis. The initial video assisted thoracoscopic anterior release and cranial bifemoral traction were uneventful (Fig. 1). Postoperatively, ten-pound weights were added at the head and another ten for counter-traction and transported to the Pediatric Intensive Care unit (PICU). Weights were added daily in 5-pound increments. Postoperative day 2, the patient complained of "double vision" which resolved upon decreasing the traction weight by 5 pounds. She underwent an uneventful posterior spinal fusion one week later under total intravenous anesthesia with propofol and remifentanil. Somatosensory and motor evoked potentials were adequate throughout the procedure in addition to a satisfactory wakeup test prior to skin closure and emergence. 


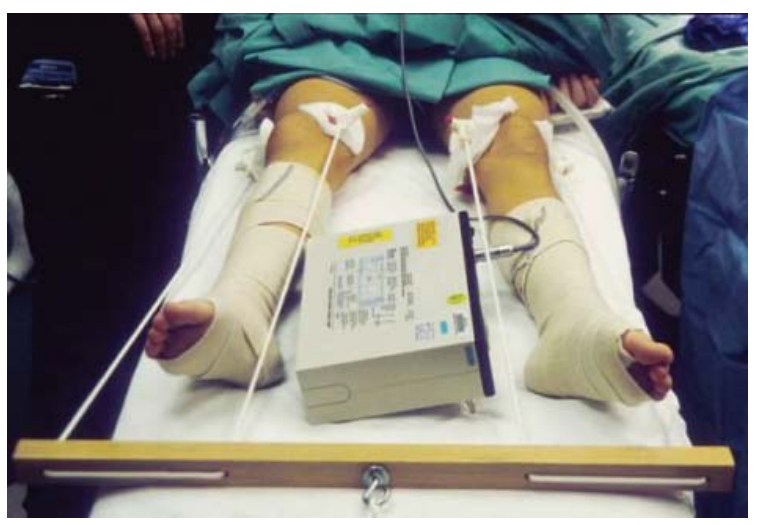

Figure 1. The femoral component of the halo-traction device. The patient is supine with the pins threaded through the femurs proximal to the condyles and attached to the weighted line.

One day later, physical examination revealed an isolated left sixth cranial nerve palsy with inability to gaze laterally. The patient did not have any other neurological deficits, and denied headaches or even double vision. Neurological and ophthalmological consultations excluded hypoxia/hypoperfusion as possible etiologies. Intraoperative blood loss was estimated to be around $6 \mathrm{~L}$ in a $53 \mathrm{~kg}$ female. However, further workup was recommended to exclude etiologies such as, meningitis, ocular myasthenia, and intracranial bleed. Magnetic resonance imaging and computerized tomography were unobtainable at the time because of the ferromagnetism in the device and metal artifact, respectively. The femoral pins and occipital frame were removed on day 3 in order to obtain imaging and also to remove a potential source of infection. The left lateral rectus palsy was noted to improve that same day and imaging results were ultimately negative.

\section{Discussion}

Scoliosis is a common deformity in children, which usually progresses into adulthood. Prevalence of spinal deformity is much higher in children with neuromuscular disorders such as cerebral palsy and Duchenne's muscular dystrophy when compared with the general population [6]. The effects of the deformity can be quite disabling and frequently require surgical correction with anterior release and posterior spinal fusion and stabilization with halo-traction.

Cranial nerve palsy secondary to halo-traction is a welldescribed phenomenon in the orthopedic literature. Patients who underwent radiation and/or with history of myelomeningocele seemed to be at most risk, however, in one report by Wilkens et al [5], the overall postoperative complication was found to be around $8.5 \%$. The common factor amongst all cranial nerve injuries was quick increases in traction, prompting some authors to recommend a maximum of 30-
$35 \mathrm{lbs}$, even in adults, and if possible to use halo-suspension traction, which uses the patient's body weight for countertraction. This method seems to have no cranial-nerve sequelae [7]. All injuries had a gradual, almost complete resolution of symptoms in 6 weeks.

Generally, abducens nerve palsy is the most common cranial nerve palsy [5], reported with different procedures such as lumbar punctures, shunt procedures, maxillary osteotomies, cranial trauma, and skull tractions. The predilection of cranial nerve VI paralysis was originally thought to be due to its long course. However, recent anatomic studies revealed multiple factors that render the nerve susceptible to injury [7]. These include multiple tethering points, a sharp turning entrance into the cavernous sinus and direct compression at the clivus. In addition to occasional penetration of anomalous labyrinthine arteries, they can also be in close proximity to the basilar and anterior inferior cerebellar arteries.

Our patient developed the palsy with only $30 \mathrm{lbs}$ traction. Moreover, symptoms resolved almost immediately upon release of the traction, in contrast to the usual slow gradual resolution described in the literature.

In conclusion, our case report demonstrates the need for frequent neurological examinations in patients in halo-traction and prompt removal of the halo traction at the first sign of problems related to the eyes.

\section{Conflict of Interest}

None

\section{References}

1. Nickel VL, Perry J, Garrett A, Heppenstall M. The halo. A spinal skeletal traction fixation device. J Bone Joint Surg Am. 1968;50(7):1400-1409.

2. Barsoum WK, Mayerson J, Bell GR. Cranial nerve palsy as a complication of operative traction. Spine (Phila $\mathrm{Pa}$ 1976). 1999;24(6):585-586.

3. Dellon AL, Campbell JN, Cornblath D. Stretch palsy of the spinal accessory nerve. Case report. J Neurosurg. 1990;72(3):500-502.

4. Kacker A, Komisar A, Kakani RS, Reich E, Rothman L. Tongue paralysis following head trauma. J Laryngol Otol. 1995;109(8):770-771.

5. Wilkins C, MacEwen GD. Cranial nerve injury from halo traction. Clin Orthop Relat Res. 1977;126:106-110.

6. Karol LA. Scoliosis in patients with Duchenne muscular dystrophy. J Bone Joint Surg Am. 2007;89 Suppl 1:155-162.

7. Hanson RA, Ghosh S, Gonzalez-Gomez I, Levy ML, Gilles FH. Abducens length and vulnerability? Neurology. 2004;62(1):33-36. 